\title{
How to look closely to vulnerable atherosclerotic plaques using nanoparticles
}

\begin{abstract}
The cardiovascular diseases (CVD) have been for several years the worldwide leading cause of death, including acute coronary syndromes (ACS) and stroke. In most cases, ACS result from erosion or rupture of a non-stenotic atherosclerotic plaques, the so called vulnerable atherosclerotic plaques. Although our knowledge about plaque vulnerability has increased over the last few years, our ability to predict the time of a future event is still highly insufficient. Different imaging technologies have been evolving to visualize various features of vulnerable plaques, though there is yet no single technique that has unequivocally demonstrated the capability to accurately predict plaque rupture in patients. Despite the great approaches for morphological imaging of atherosclerotic plaques, the need for a molecular profile of an atheroma clearly emerges. In this short review we pretend to discuss the most commonly used diagnostic tools in CHD and how the combination with nanoparticles can bring these approaches to the molecular imaging level. We highlight the approaches with higher technology readiness level from invasive and non-invasive imaging approaches
\end{abstract}

Keywords: Acute coronary syndrome, Vulnerable atherosclerotic plaque, Intravascular imaging, Gold nanoparticles
Volume 6 Issue I - 2017

\author{
Catia Costa Oliveira,' Marina Brito ${ }^{2}$ \\ 'Life and Health Sciences Research Institute (ICVS), University \\ of Minho, Portugal \\ International Iberian Nanotechnology Laboratory, Portugal
}

Correspondence: Marina Brito, International Iberian Nanotechnology Laboratory, Braga, Portugal, Email vmarina.brito@inl.int

Received: July 10, 2017 | Published: August 30, 2017
Abbreviations: CHD, Coronary Heart Disease; ACS, Acute Coronary Syndrome; IVUS, Intravascular Ultrasonography; NIRS, Near-Infrared Spectroscopy; MRI, Magnetic Resonance Imaging; OCT, Optical Coherence Tomography; USPIO, Ultrasmall Supermagnetic Particles of Iron Oxide; AuNPs, Gold Nanoparticles

\section{Introduction}

Coronary heart disease (CHD) continues to be a major cause of morbidity and mortality throughout the world.$^{1}$ Nearly two thirds of myocardial infarctions occur due to rupture and thrombosis of nonflow-limiting plaques in lesions that showed only moderate stenosis. The risk of rupture in nonstenotic plaques seems to depend more on plaque composition rather than plaque size or fibrous cap thickness solely. The vulnerable plaques are morphologically characterized by outward remodeling, increased plaque neovascularity, intraplaque hemorrhage, smooth muscle cell depletion and microcalcification. It is also descried that namely intimal and adventitial inflammation play a key role in plaque vulnerability, rupture and subsequent thrombosis, exposing that the plaque instability is intrinsically related to the atherosclerotic inflammatory process .,3 Detection of a vulnerable plaque and subsequent rupture prevention is a remaining challenge in cardiology. Mostly because the available diagnostic tools lack the molecular information and it is now clear that based in purely anatomic imaging it is difficult to predict an ACS . ${ }^{4}$ Among the available diagnostic tools the intravascular imaging and molecular imaging probes are emerging as possible methods for this purpose, though none is available for accurately predict plaque rupture. In this mini-review we will focus on the most promising imaging tools combinations that can finally deliver a complete diagnose of plaque vulnerability.

\section{Discussion}

Among the non-invasive techniques, the coronary angiography is the commonly first approach for medical doctor for Coronary Heart Disease (CHD) diagnosis. It allows the detection of lumen stenosis and thrombus with a high spatial resolution. However, it does not have enough resolution to evaluate the artery wall and, thus it may not identify the presence of a vulnerable plaque. ${ }^{5}$ Another non-invasive imaging approach is the MRI (Magnetic Resonance Imaging), widely explored in different medical areas as a powerful diagnostic tool. Regarding the cardiovascular area, the MRI is known to deliver temporal and spatial resolution adequate for studies on larger arteries as aorta and carotid arteries, although even the cutting-edge devices, do not own enough resolution to distinguish the coronary atherosclerotic stenosis and composition $.5,6$ In this scenario, intravascular modalities, with their ability to go beyond the detection of lumen stenosis and assess to visual features of vulnerable plaques, can add significant information about the disease. Intravascular ultrasonography (IVUS) is widely available and safe. It has the capacity of real-time cross-sectional assessment of lumen dimension and plaque morphology with a depth penetration superior to $5 \mathrm{~mm}$ with robust measurements. ${ }^{7}$ However conventional IVUS does not possess enough sensibility for accurate detection of the lipid pool, a hallmark of vulnerability. To overcome this lack of resolution of conventional IVUS, the Virtual Histology-IVUS (VH-IVUS) was developed using advanced radiofrequency analyses of reflected echo signals to generate multiple spectral analyses. VH-IVUS has a high sensitivity on identification of lipid-rich plaque, a key feature of a vulnerable plaque.$^{7}$ Ex vivo studies demonstrated that VH-IVUS provides accurate details on coronary plaque composition with high agreement with the histology. Moreover this approach was performed in the PROSPECT study, as imaging technique to assess vulnerable plaques ${ }^{8}$ The PROSPECT trial - a prospective multicenter study of the natural history of coronary atherosclerosis - showed that events unrelated to non-culprit lesions were associated with thin-cap fibroatheromas based on VH-IVUS assessment, which is consistent with the concept of the vulnerable plaque. Though these findings are promising, the criteria used in VH-IVUS in the PROSPECT to identify these plaques lack specificity and only predicted $18 \%$ of coronary events after three years.$^{8}$ Another widely used intravascular imaging approach is the Near-Infrared Spectroscopy (NIRS), which has been 
applied in the coronary circulation mainly for accurate lipid content and necrotic core detection . ${ }^{9}$ In order to overcome the lack of structural information with NIRS, researchers have combined two techniques - NIRS with IVUS - and delivered a high potential new tool that allows the identification and morphometric assessment of lipid-rich plaques together with the synergistic value of plaque volumetric and compositional imaging. This combination is, nowadays, one of the most accurate diagnostic tool for unstable plaques.$^{10}$ The Optical Coherence Tomography (OCT), a widespread technology in medicine, commonly used in ophthalmology for a long time and more recently in cardiology, where it immediately became an advantageous alternative to the other conventional technology. The results from this imaging tool resembles IVUS, although its principle is based on near-infrared broad band light sources to scan tissues instead of ultrasound.${ }^{11}$ OCT has 10 -fold higher spatial resolution than IVUS which permits the assessment to different parameters for plaque vulnerability classification: fibrous cap length, limited identification of macrophage distribution through the plaque and neovascularization. Some authors state that OCT is an ideal method for vulnerable plaque detection, mostly because OCT showed a better performance on the identification of fibrous cap disruption and erosion, intracoronary thrombus and thin-cap fibroatheroma than IVUS.$^{12,13}$ In addition, a study in which the ability of in vivo lipid pool assessment was evaluated, demonstrated the superiority of OCT when compared to IVUS, since IVUS showed only $24.1 \%$ of OCT sensitivity for this purpose. Importantly, it is also proven that there is a concordance between OCT and histological measurements regarding the fibrous cap length in human cadavers, which is a great feature of plaque vulnerability.${ }^{14}$ Still, due to its limited penetration $(1-2 \mathrm{~mm})$, it may not detect larger plaques as well as the total plaque burden. Though it can provide morphological information regarding the lesion, OCT alone does not provides molecular and biochemical specific information of the atherosclerotic plaques without the administration of a contrast agent.$^{15}$ Different labs in the world are now working on development of a contrast agent that can signal molecular mechanisms.$^{15}$ The introduction of exogenous contrast agent will provide to OCT subcellular imaging information and, consequently, it will allow to distinguish different states of plaque progression. Along with the optimization of the imaging techniques, the imaging diagnosis approach has evolved by adding new exogenous contrast agents. Early on micro and nanoparticles have become very interesting candidates to enhance inflammation signals, because it was showed that particles, as USPIO, are eliminated by phagocytosis by the monocyte-macrophage cell system.$^{16,17}$ Atherosclerosis is known to have high inflammation activity, thus USPIO could be used to enhance MRI imaging of atherosclerotic plaques. With this in mind, in 2001 Ruehm and colleagues have presented a complete study proving that USPIO can outline the inflammatory changes along the atherosclerotic disease process in hyperlipidemic rabbits due to the accumulation of these particles in the macrophages inside atherosclerotic plaques.$^{18}$ Different studies have been done in humans using USPIO.$^{19,20}$ Clinical studies using USPIO have already been performed and confirmed USPIO accumulation in macrophages of ruptured and rupture-prone human carotid atherosclerotic lesions in MRI images ..$^{21-23}$ Though USPIO-enhanced MRI of atherosclerotic plaques demonstrate potential as a noninvasive technique for imaging inflammatory activity in plaques as the aorta or even carotid, it cannot be use for coronary arteries imaging due to the MRI resolution limitation [6]. Gold Nanoparticles (AuNPs), on the other hand, present unique optical features that make this a viable contrast agent for OCT. Their detection relies on the local surface plasmon resonance effect, caused by the incident OCT light and it depends on the relative change in the backscattering coefficient between the NP and the medium (as in highly scattering media NP with low scattering cross section lead to a maximum OCT contrast).$^{24}$ Recently, a study of dynamic single gold nanoparticle visualization by intracoronary OCT clearly demonstrated that AuNPs presence induced a relevant enhancement in the OCT contrast. This study showed that OCT signal has a linear increase with the back-scattering cross section given by each AuNPs, which is highly dependent on the particular type of AuNPs used.$^{25} \mathrm{Hu}$ and colleagues clearly demonstrated that, for the same concentration, gold nanoshells (multipolar plasmonic excitations in the 1000-1500 $\mathrm{nm}$ spectral range) present a higher contrast when compared to gold nanorods, showed by their extinction cross section values at OCT operating wavelength more than two orders of magnitude larger than gold nanorods, together with a reduced absorption efficiencies ${ }^{25}$ With this study, it is now proved the potential ability of intravascular OCT for the detection on individual AuNPs in both static and non-static conditions and it opened new applications of this combination as real time evaluation of intravascular obstruction or pressure gradients. Regarding concerns with toxic effects of nanoparticles, different toxicological studies reported excellent biocompatibility and low cytotoxicity of AuNPs.$^{26,27}$ Additionally, other studies demonstrated that the surface of AuNPs can be easily functionalized to contain molecular and organelle-targeting ligands and peptides.$^{28}$ Therefore, functionalized AuNPs may be used for imaging applications of a single cell (for instances to differentiate between an abnormal cell, as a neoplasic cell, and normal cell), to molecular imaging strategies and to targeted delivery of specific molecules as drugs . ${ }^{29,30}$ Taken together all the mentioned works, it is clear that AuNPs can be functionalized with antibodies for targeted recognition of biomarkers for plaque vulnerability, and this way enhancing OCT ability to characterize vulnerable plaques.

\section{Conclusion}

There is a close relationship between plaque vulnerability and patient outcome which pushes researchers to unveiled new approaches to image the different atheroma features. From all the available imaging techniques, the invasive modalities offer the highest spatial resolution. OCT is a well clinical established technique for atherosclerotic heart disease's evaluation. The addition of contrast agents, namely nano-probes that specifically recognize vulnerability biomarkers in atheromas, will improve the current diagnostic capabilities, resulting in early recognition and prognosis of artery diseases with high-risk features, a crucial step to identify those patients who will likely develop acute events in the near future. Although, nanosized particles had already showed the ability to act as efficient contrast agents for OCT, selective molecular identification of inflammation processes using OCT imaging mediated by nanoparticles still must be unraveled.

\section{Conflicts of interest}

The authors declare no conflict of interest.

\section{Acknowledgments}

None.

\section{References}

1. WHO. Top 10 causes of death. 2017

2. Finn AV, Nakano M, Narula J, et al. Concept of Vulnerable/Unstable Plaque. Arterioscler Thromb Vasc Biol. 2010;30(7):1282-1292.

3. Shah PK. Biomarkers of Plaque Instability. Curr Cardiol Rep. 2014;16(12):1-8. 
4. Majmudar MD, Nahrendorf M. Cardiovascular Molecular Imaging: The Road Ahead. J Nucl Med. 2012;53(5):673-676.

5. Celeng C, Takx RAP, Ferencik M, et al. Non-invasive and invasive imaging of vulnerable coronary plaque. Trends Cardiovasc Med. 2016;26(6):538-547.

6. Marques JS, Pinto FJ, Marques JS, et al. The vulnerable plaque: Current concepts and future perspectives on coronary morphology, composition and wall stress imaging. Rev Port Cardiol. 2016;33(2):101-110.

7. Nair A, Kuban BD, Tuzcu EM, et al. Coronary Plaque Classification With Intravascular Ultrasound Radiofrequency Data Analysis. Circulation. 2002;106(17):2200-2206.

8. Bruyne B De, Cristea E, Mintz GS, et al. A Prospective Natural-History Study of Coronary Atherosclerosis. 2011.

9. Psaltis PJ, Nicholls SJ. Imaging: Focusing light on the vulnerable plaque. Nat Rev Cardiol. 2016;13(5):253-255.

10. Puri R, Madder RD, Madden SP, et al. Near-Infrared Spectroscopy Enhances Intravascular Ultrasound Assessment of Vulnerable Coronary Plaque. Arterioscler Thromb Vasc Biol. 2015;35(11):2423-2431

11. Braz AKS, Araujo RE De, Ohulchanskyy TY, et al. In situ gold nanoparticles formation: contrast agent for dental optical coherence tomography for dental optical coherence tomography. $J$ Biomed Opt. 2012;17(6):066003.

12. Kubo T, Tanaka A, Ino Y, et al. Assessment of Coronary Atherosclerosis using Optical Coherence Tomography. $J$ Atheroscler Thromb. 2014;21(9):895-903.

13. Kubo T, Maehara A, Mintz GS et al. The Dynamic Nature of Coronary Artery Lesion Morphology Assessed by Serial Virtual Histology Intravascular Ultrasound Tissue Characterization. J Am Coll Cardiol 2010;55(15):1590-1597.

14. Kume T, Akasaka T, Kawamoto T, et al. Measurement of the thickness of the fibrous cap by optical coherence tomography. Am Heart $J$. 2006;152(4):755.e1-4.

15. Boppart SA, Oldenburg AL, Marks DL Optical probes and techniques for molecular contrast enhancement in coherence imaging. 2015;10(4):41208.

16. Raynal I, Prigent P, Peyramaure S et al. Macrophage Endocytosis of Superparamagnetic Iron Oxide Nanoparticles: Mechanisms and Comparison of Ferumoxides and Ferumoxtran-10. Invest Radiol. 2004;39(1):56-63.

17. Metz S, Bonaterra G, Rudelius M et al. Capacity of human monocytes to phagocytose approved iron oxide MR contrast agents in vitro. Eur Radiol. 2004;14(10):1851-1858.
18. Ruehm SG, Corot C, Vogt $\mathrm{P}$ et al. Magnetic Resonance Imaging of Atherosclerotic Plaque with Ultrasmall Superparamagnetic Particles of Iron Oxide in Hyperlipidemic Rabbits. Circulation. 2001;103(3):415-422.

19. Schmitz SA, Taupitz M, Wagner S et al. Magnetic resonance imaging of atherosclerotic plaques using superparamagnetic iron oxide particles. $J$ Magn Reson Imaging. 2001;14(4):355-361.

20. Usman A, Sadat U, Patterson AJ et al. Use of ultrasmall superparamagnetic iron oxide particles for imaging carotid atherosclerosis. Nanomed. 2015;10(19):3077-3087.

21. Kooi ME, Cappendijk VC, Cleutjens KBJM et al. Accumulation of Ultrasmall Superparamagnetic Particles of Iron Oxide in Human Atherosclerotic Plaques Can Be Detected by In Vivo Magnetic Resonance Imaging. Circulation. 2003;107(19):2453-2458.

22. Tang TY, Howarth SPS, Miller SR et al. The ATHEROMA (Atorvastatin Therapy: Effects on Reduction of Macrophage Activity) Study: Evaluation Using Ultrasmall Superparamagnetic Iron Oxide-Enhanced Magnetic Resonance Imaging in Carotid Disease. J Am Coll Cardiol. 2009;53(22):2039-2050.

23. Trivedi RA, Mallawarachi C, U King Im JM et al. Identifying Inflamed Carotid Plaques Using In Vivo USPIO-Enhanced MR Imaging to Label Plaque Macrophages. Arterioscler Thromb Vasc Biol. 2006;26(7):1601-1606.

24. Austin LA, Kang B, El-Sayed MA Probing molecular cell event dynamics at the single-cell level with targeted plasmonic gold nanoparticles: A review. Nano Today. 2015;10(5):542-558.

25. Hu J, Rivero F, Torres RA et al. Dynamic single gold nanoparticle visualization by clinical intracoronary optical coherence tomography. $J$ Biophotonics. 2017;10(5):674-682.

26. Connor EE, Mwamuka J, Gole A et al.Gold Nanoparticles Are Taken Up by Human Cells but Do Not Cause Acute Cytotoxicity. Small. 2005;1(3):325-327.

27. Murphy CJ, Gole AM, Stone JW et al. Gold Nanoparticles in Biology: Beyond Toxicity to Cellular Imaging. Acc Chem Res. 2008;41(12):1721-1730.

28. Leduc C, Si S, Gautier J et al. A Highly Specific Gold Nanoprobe for Live-Cell Single-Molecule Imaging. Nano Lett. 2013;13(4):1489-1494.

29. Kim D, Jeong YY, Jon S A Drug-Loaded Aptamer-Gold Nanoparticle Bioconjugate for Combined CT Imaging and Therapy of Prostate Cancer. ACS Nano. 2010;4(7):3689-3696.

30. Lee SM, Kim HJ, Ha YJ et al. Targeted Chemo-Photothermal Treatments of Rheumatoid Arthritis Using Gold Half-Shell Multifunctional Nanoparticles. ACS Nano. 2013;7(1):50-57. 